\title{
Prevalencia, factores de riesgo y manejo de la depresión en pacientes con infección por VIH: Revisión de la literatura
}

\author{
Claudia Wolff L., Rubén Alvarado M. y Marcelo Wolff R.
}

\section{Depression in HIV infection: \\ Prevalence, risk factors and management}

Depression is one of the main psychiatric co-morbidities in HIV infection, presenting with a significantly higher prevalence than in the general population (around 35\%). Its presence has been associated with poor quality of life, HIV disease progression and poor adherence to antiretroviral therapy. Although antidepressive treatment has demonstrated effectiveness on the management of depressive symptoms, improvement of clinical and laboratory parameters, and enhancement of antiretroviral adherence, depression is frequently under diagnosed and under treated in these patients. We analyzed the main international findings on depression prevalence, risk factors, consequences and management in people with HIV disease.

Key words: HIV, AIDS, depression, antidepressive treatment.

Palabras clave: VIH, SIDA, depresión, tratamiento antidepresivo.

\section{Introducción}

$\mathrm{L}$ a depresión es muy frecuente entre los pacientes con enfermedades crónicas, caracterizándose por presentar el mayor sub-registro y sub-tratamiento dentro de las co-morbilidades psiquiátricas ${ }^{1,2}$. En el caso de los pacientes infectados por virus de inmunodeficiencia humana (VIH), la depresión suele ser dos a cuatro veces más frecuente que en la población general, considerándose como una de las co-morbilidades psiquiátricas más frecuentes y la de mayor incidencia tras el diagnóstico de la infección ${ }^{1,3,4}$. A nivel internacional existe amplia referencia en la literatura biomédica respecto a la prevalencia de depresión, sus factores de riesgo y consecuencias asociadas en pacientes con infección por VIH. Sin embargo, en Chile no hay investigaciones publicadas al respecto. En este trabajo se revisarán los principales hallazgos provenientes de estudios realizados en el extranjero en relación a la depresión en pacientes infectados por VIH, con el propósito de proveer información al médico clínico $\mathrm{y}$ al resto de los profesionales de la salud que trabajan con estas personas y así contribuir al logro de mejores resultados

\section{Material y Métodos}

Esta revisión está basada en una selección de artículos publicados en las bases de datos PubMed, y scielo, uti- lizando como palabras claves "VIH", "SIDA" asociados a "depresión", "síndrome depresivo" y "trastornos psiquiátricos", así como "psicofarmacología" y tratamiento antidepresivo. Asimismo, se realizó una búsqueda combinando "terapia anti-retroviral (TARV)" con "complicaciones neuropsiquiátricas”. La búsqueda se limitó a artículos en inglés y español, publicados entre enero de 1995 y enero de 2009. Se seleccionaron 49 publicaciones, que a juicio de los autores eran las más relevantes, sin utilizar criterios de selección específicos.

\section{Prevalencia de episodios depresivos}

La prevalencia de depresión en pacientes con infección por VIH se describe en torno al $35 \%$, pero existe gran variabilidad en el reporte, con valores que fluctúan entre 20 y 79\%, dependiendo de la población estudiada, del periodo de tiempo investigado, así como de los instrumentos utilizados para la definición de caso ${ }^{2-7}$. En la Tabla 1 se presentan las prevalencias encontradas en diferentes publicaciones. En E.U.A., en una muestra de pacientes infectados por VIH en control, con representatividad nacional, se encontró una prevalencia de depresión de 36\% durante el último año ${ }^{5}$. En América Latina, los reportes provienen de Brasil, donde Noqueira y cols ${ }^{8}$, y Antakly de Melo y Malgebier ${ }^{9}$, observaron una prevalencia puntual de depresión de $21,8 \%$ en una muestra mixta y de $25,8 \%$ en una muestra compuesta sólo por mujeres, respectivamente.

\author{
Facultad de Medicina, \\ Universidad de Chile, \\ Santiago \\ Escuela de Salud \\ Pública (CWL, RAM) \\ Departamento de Medicina-Campus \\ Centro (MWR). \\ Universidad Mayor, Santiago, \\ Chile (CWL) \\ Programa de formación \\ en Psiquiatría \\ Servicio de Salud \\ Metropolitano Central, \\ Santiago, Chile \\ Complejo de Salud San Borja \\ Arriarán Fundación Arriarán (MWR)
}

Recibido: 7 de junio de 2009

Aceptado: 10 de diciembre de 2009

Correspondencia a:

Claudia Wolff Levi

clausiwola@msn.com 


\begin{tabular}{|c|c|c|c|c|c|}
\hline Investigadores & $\begin{array}{c}\text { Año } \\
\text { publicación }\end{array}$ & Instrumento utilizado & Población estudiada & Tamaño muestral & Prevalencia depresión \\
\hline Pao M. et al' ${ }^{11}$ & 2000 & - SCID-P & Adolescentes & 34 & $44 \%$ (puntual) \\
\hline Morrisson M. et al ${ }^{2}$ & 2002 & $\begin{array}{l}\text { - SCID DSM IV } \\
\text { - HAM-D } \\
\text { - HAM-A }\end{array}$ & Mujeres VIH+NIH- & $\begin{array}{l}93 \mathrm{VIH}+ \\
62 \mathrm{VIH}-\end{array}$ & $\begin{array}{l}-19,1 \text { (puntual) y } 47,3 \% \\
\text { (de vida) VIH+ } \\
-4,8 \% \text { (puntual) y } 38.7 \% \\
\text { (de vida) VIH- }\end{array}$ \\
\hline Asch S. et al ${ }^{1}$ & 2003 & $\begin{array}{l}- \text { CIDI-SF } \\
\text { - CIDI }\end{array}$ & Ambos sexos & 1.140 & $37 \%$ (anual) \\
\hline Turner B. et al ${ }^{21}$ & 2003 & $\begin{array}{l}\text { Revisión de prescripciones médi- } \\
\text { cas por antidepresivos }\end{array}$ & Ambos sexos, usuarios de drogas & $\begin{array}{l}\text { 1.827 Mujeres } \\
3.246 \text { Hombres }\end{array}$ & $\begin{array}{l}\text { - } 34 \% \text { Mujeres } \\
\text { - 29\% Hombres (puntual) }\end{array}$ \\
\hline Cook J. et al ${ }^{22}$ & 2004 & CES-D & Mujeres & 1.716 & $\begin{array}{l}-32 \% \text { (puntual) } \\
-35 \% \text { (de vida) }\end{array}$ \\
\hline Bouhnik M. et al56 & 2005 & - CES-D & Ambos sexos, usuarios de drogas & 243 & $46 \%$ (puntual) \\
\hline Weiser S. et $\mathrm{al}^{3}$ & 2006 & $-B D I$ & Hombres en situación de calle & 239 & $56 \%$ (puntual) \\
\hline Noqueira et $a^{8}$ & 2006 & - HAD & Ambos sexos & 386 & $21,8 \%$ (puntual) \\
\hline $\begin{array}{l}\text { Antakly de Mello V., } \\
\text { Malgebier A. }{ }^{9}\end{array}$ & 2006 & $\begin{array}{l}\text { - SCID-DSM IV } \\
\text { - HAM-D } \\
\text { - BDI }\end{array}$ & Mujeres & $\begin{array}{l}\text { - } 60 \text { sintomáticas } \\
\text { - } 60 \text { asintomáticas }\end{array}$ & $25,8 \%$ (puntual) \\
\hline Yi M. et al ${ }^{25}$ & 2006 & CESD-10 & Ambos sexos & 450 & $53,6 \%$ (puntual) \\
\hline $\begin{array}{l}\text { Turner B., } \\
\text { Fleishman J.13 }\end{array}$ & 2006 & CIDI-SF & Ambos sexos & 1.982 & $\begin{array}{l}35 \% \text { Mujeres } \\
31 \% \text { Hombres (anual) }\end{array}$ \\
\hline Pence B. et $a l^{6}$ & 2006 & - SCID-DSM IV & Ambos sexos & 1.125 & $30 \%$ (anual) \\
\hline Jin $\mathrm{H}$. et $\mathrm{al}^{7}$ & 2006 & $\begin{array}{l}-\mathrm{CIDI} \\
-\mathrm{BDI}\end{array}$ & Ambos sexos & $\begin{array}{l}28 \mathrm{VIH}+ \\
23 \mathrm{VIH}-\end{array}$ & $79 \%$ (de vida) \\
\hline Israelski D. et al'12 & 2007 & $-\mathrm{BDI}$ & Ambos sexos & 210 & $38 \%$ (puntual) \\
\hline Lima V. et al ${ }^{27}$ & 2007 & - CES-D & Ambos sexos & 563 & $51 \%$ (puntual) \\
\hline \multicolumn{6}{|c|}{$\begin{array}{l}\text { BDI: Inventario de Depresión de Beck. CES-D: Escala de depresión del Centro de Estudios Epidemiológicos. CESD-10: Escala de Depresión del Centro de Estudios Epidemioló } \\
\text { gicos- } 10 \text { ítems. CIDI: Composite International Diagnostic Interview. CIDI-SF: Composite International Diagnostic Interview- Short form. HAD: Escala hospitalaria de ansiedad } \\
\text { depresión. HAM-A: Escala de ansiedad de Hamilton. HAM-D: Escala de depresión de Hamilton. SCID-DSM IV: Entrevista clínica estructurada para trastornos del Eje I del DSN } \\
\text { IV. SCID-P: Entrevista clínica estructurada - edición para pacientes. }\end{array}$} \\
\hline
\end{tabular}

Cuántos de los episodios depresivos que se presentan en los pacientes con infección por VIH corresponden a un primer y único episodio, y cuántos se enmarcan dentro de un trastorno del ánimo crónico es un tema poco estudiado, ya que la mayoría de las investigaciones se han abocado a la estimación de la prevalencia puntual y/o de último año, y no a la prevalencia de vida. Asimismo existen escasos reportes respecto a la prevalencia de depresión antes y después de contraer el VIH. Algunos estudios que apuntan en esta dirección son los de Jin y cols. en China, que reportaron $79 \%$ de trastorno depresivo crónico (con tan sólo un caso diagnosticado antes de contraer el VIH) ${ }^{7}$ y el de Gibbie y cols, en Australia, en el que encontraron 39 y $15 \%$ de antecedentes de depresión pasada en pacientes con infección por VIH y en controles seronegativos, respectivamente ${ }^{10}$. Morrisson y cols, compararon mujeres con infección por VIH con controles seronegativos, encontrando una prevalencia puntual de depresión de 19,1 y $4,8 \%$ y de 47,3 y $38,7 \%$ de prevalencia de vida, respectivamente ${ }^{2}$.

La prevalencia de trastornos psiquiátricos en general, no sólo de depresión en particular, es también alta en la población portadora del VIH, con reportes que fluctúan entre 38 y $85 \%$ 5,6,11,12. En un estudio con representatividad nacional realizado en población VIH en E.U.A., cerca de $50 \%$ de los individuos presentaron criterios para algún trastorno psiquiátrico. Un 36\% mostró signos de depresión en las pruebas de tamizaje, $26,5 \%$ para distimia, $15,8 \%$ 
para el trastorno de ansiedad generalizado, 10,5\% para el trastorno de pánico y $12 \%$ para la dependencia de sustancias en los últimos 12 meses $^{5}$. En otros subgrupos estudiados se han reportado prevalencias de $40 \%$ para los trastornos ansiosos, 35\% para los trastornos distímicos, $54 \%$ para la ideación e intento suicida, y de 14 a $43 \%$ para trastornos por estrés postraumático ${ }^{13-17}$. Muchos de estos trastornos co-existen con los episodios depresivos. Pao y cols, reportaron que en su muestra de pacientes adolescentes infectados, $44 \%$ se encontraba deprimido y que de éstos $40 \%$ consumía concomitantemente alcohol y/o drogas ${ }^{11}$. Pence y cols, estudiaron una población con infección por VIH del sudeste de E.U.A., encontrando $8 \%$ de pacientes con criterios tanto para depresión como para abuso de sustancias ${ }^{6}$. Israelski y cols, por su parte, en un estudio que evaluaba la presencia de depresión, estrés post traumático y estrés agudo en pacientes seropositivos, encontraron que existía $38 \%$ de individuos con al menos dos de estos diagnósticos ${ }^{12}$.

\section{Características clínicas del cuadro depresivo en pacientes con infección por $\mathrm{VIH}$}

Los episodios depresivos en pacientes seropositivos para VIH, en general comparten las mismas características clínicas que en pacientes seronegativos. Los pacientes también manifiestan desánimo y/o anhedonia, pueden presentar cambios conductuales tales como aislamiento e irritabilidad y están expuestos a sufrir los cambios neurovegetativos propios de este trastorno, tales como insomnio, inapetencia, polifagia y cambios en el peso corporal $^{18,19}$. De igual forma, pueden presentar toda la gama de alteraciones neuro-cognitivas propias de los cuadros depresivos, tales como dificultad de concentración, falla en la memoria y enlentecimiento psicomotor ${ }^{18}$. Aparentemente, la principal diferencia estaría dada por la mayor frecuencia e intensidad de los síntomas entre los pacientes infectados por VIH, especialmente entre las mujeres ${ }^{2}$. El diagnóstico de depresión puede no ser fácil, por lo que se han establecido criterios diagnósticos consensuados que son aplicables tanto a población general, como a población con morbilidad, incluidos los pacientes seropositivos para VIH. En la Tabla 2 se exponen los criterios diagnósticos para un episodio depresivo según el Manual Diagnóstico y Estadístico de los Trastornos Mentales de la Sociedad Americana de Psiquiatría (DSM), versión IV, que corresponde a uno de los más utilizados actualmente a nivel mundial ${ }^{20}$.

\section{Factores asociados}

Al igual a lo descrito para la población general, los pacientes con mayor riesgo de desarrollar depresión son los de sexo femenino, aquellos con menor nivel educacional y socioeconómico, los desempleados o en condiciones de inestabilidad laboral, los no casados ni emparejados, y aquellos que no cuentan con una red de apoyo social ${ }^{2,4,5,8,9,21-25}$. Junto a esto, se considera que los pacientes con mayores preocupaciones respecto a su situación de salud, con mayor incomodidad respecto a la forma en que contrajeron el VIH, así como con menos creencias religiosas o espirituales, presentarían mayor vulnerabilidad a la depresión ${ }^{11,24,25}$. Otro grupo particularmente vulnerable a desarrollar depresión sería el de los hombres homosexuales, aunque algunos estudios indican que la prevalencia de depresión entre éstos sería la misma que para los hombres homosexuales seronega$\operatorname{tivos}^{2,4}$. Se ha descrito que los adolescentes seropositivos tendrían hasta cuatro veces más riesgo que sus pares de la población general de desarrollar depresión y que este riesgo superaría el encontrado en adolescentes con otras enfermedades crónicas, tales como diabetes mellitus o cáncer ${ }^{11}$.

En cuanto a la sintomatología, se ha visto que los pacientes con mayor cantidad y severidad de los síntomas atribuibles a la infección, presentarían mayor riesgo de depresión ${ }^{2,9,21,26,27}$. Antakly de Mello y Malgebier, en un estudio realizado en mujeres, encontraron que la prevalencia de depresión variaba si se subdividía a su muestra en un grupo con síntomas asociados al VIH y

\section{Tabla 2. Criterios para el episodio depresivo mayor según el DSM-IV}

A. Presencia de cinco (o más) de los siguientes síntomas durante al menos dos semanas, que presentan un cambio respecto a la actividad previo; uno de los síntomas de ser (1) estado de ánimo depresivo o (2) pérdida de interés o de la capacidad de placer:

- Estado de ánimo depresivo la mayor parte del día, casi cada día según lo indica el propio sujeto o la observación realizada por otros

- Disminución acusada del interés o la capacidad para el placer en todas o casi todas las actividades, la mayor parte del día, casi día a día (según refiere el propio sujeto u observan los demás)

- Pérdida importante de peso sin hacer régimen, o aumento de peso, o pérdida o aumento del apetito casi cada día

- Insomnio o hipersomnia casi cada día

- Agitación o enlentecimiento psicomotor casi cada día (observable por los demás, no meras sensaciones de inquietud o de estar enlentecido)

- Fatiga o pérdida de energía casi cada día

Sentimientos de inutilidad o culpa excesivos o inapropiados (que pueden ser delirantes) casi cada día (no los simples autoreproches o culpabilidad por el hecho de estar enfermo)

- Disminución de la capacidad de pensar o concentrarse, o indecisión, casi cada día (ya sea una atribución subjetiva o una observación ajena)

- Pensamientos recurrentes de muerte (no sólo temor a la muerte), ideación suicida recurrente sin un plan específico, o una tentativa o un plan específico para suicidarse

B. Los síntomas no cumplen criterios para un episodio mixto

C. Los síntomas provocan malestar clínicamente significativo o deterioro social, laboral o de otras áreas importantes de la actividad del individuo

D. Los síntomas no son debidos a los efectos fisiológicos directos de una sustancia (droga o medicamento), o una enfermedad médica

E. Los síntomas no se explican mejor por la presencia de un duelo, los síntomas persisten durante más de dos meses, se caracterizan por una acusada incapacidad funcional, preocupaciones mórbidas de inutilidad, ideación suicida, síntomas psicóticos o enlentecimiento psicomotor 
otro asintomático. La prevalencia del primero alcanzaba a $38,3 \%$, versus $13,3 \%$ en el segundo, diferencia que fue estadísticamente significativa. Adicionalmente, en el primer grupo los síntomas depresivos fueron más graves ${ }^{9}$.

Por otra parte, se ha reportado que la depresión en sí puede afectar negativamente la evolución de la infección por VIH, con peor estado inmunológico expresado en un menor recuento de linfocitos T CD4+, mayor carga viral, progresión de la enfermedad y mayor mortalidad ${ }^{4,5,18,27-32}$. En las mujeres, la mayor mortalidad no dependería exclusivamente de condiciones directamente asociadas al VIH sino también de una mayor frecuencia de accidentes, actos de violencia y sobredosis de alcohol y drogas $2,22,28$. Esto también se refleja en el hecho de que los pacientes con infección por VIH deprimidos hacen un uso significativamente mayor de los sistemas de salud que sus pares no deprimidos ${ }^{31}$.

Los avances en TARV han sido notables, con marcada reducción de la morbi-mortalidad ${ }^{26,32,33}$. Al respecto, una condición básica para este éxito terapéutico, compartida por muy pocas otras enfermedades, es un cumplimiento extremo de la indicación medicamentosa (adherencia). Pequeños incumplimientos de esta adherencia, se asocian a fracaso terapéutico (pérdida de la capacidad de suprimir la replicación viral con la consiguiente progresión de la enfermedad), agravado por la pérdida de efectividad de los anti-retrovirales previamente usados (desarrollo de resistencia $)^{29,34-36}$. Entre los factores psico-sociales más importantes para la pérdida de adherencia están: la falta de una red de apoyo social, el abuso de sustancias y la depresión ${ }^{23,28,29,34-39}$. Se considera que la depresión no tratada es, a su vez, un factor de riesgo para el abuso de sustancias y el uso de terapias alternativas o la automedicación, con el subsecuente perjuicio en la adherencia y efectividad de la $\mathrm{TAR}^{4,11,18,28,31,32}$. Por otro lado, se ha reportado que los pacientes deprimidos estarían más vulnerables a involucrarse en conductas sexuales peligrosas, con el eventual riesgo de adquisición de otras enfermedades de transmisión sexual y contagio del VIH a terceros ${ }^{11,18,26,28,31}$.

\section{Manejo}

En la práctica clínica, la depresión en los pacientes con infección por VIH suele ser sub-diagnosticada y subtratada $1,31,32,40$. Asch y cols, encontraron que entre estos pacientes existía un sub-diagnóstico de depresión de 45\%, que afectaba principalmente a los pacientes con menor nivel educacional y que, interesantemente, los médicos que más sub-diagnosticaban eran aquellos que atendían mayor número de pacientes infectados por $\mathrm{VIH}^{1}$. Antakly de Mello y Malgebier, encontraron que a pesar de la alta prevalencia de depresión que existía en su muestra, tan sólo $11 \%$ de las pacientes estaba en tratamiento antidepresivo ${ }^{9}$. Israelski y cols, en su estudio sobre prevalencia de depresión y trastornos por estrés, encontraron tan sólo
$49 \%$ de pacientes en tratamiento, mientras que Jin y cols, encontraron que de $79 \%$ de pacientes que presentaban una depresión, tan solo $9 \%$ recibía antidepresivos ${ }^{7,12}$. Las razones por las que la depresión es sub-diagnosticada y sub-tratada en estos individuos son variadas, y van desde aspectos prácticos como la falta de tiempo de los médicos para evaluar los problemas psiquiátricos y psicológicos de sus pacientes durante la consulta médica, hasta aspectos relacionados con la falta de formación en la pesquisa de depresión y/o la presencia de prejuicios e ideas preconcebidas en el área de salud mental por parte del personal de salud ajeno a ésta ${ }^{1,18,41}$. Se considera que los equipos de salud tienden inadecuadamente a considerar como normal y esperable la sintomatología depresiva en estos pacientes o que en presencia de complicaciones con riesgo vital los síntomas depresivos no son trascendentes o de urgencia terapéutica ${ }^{1,18,41}$. Por lo mismo, muchos profesionales no indagarían en la sintomatología depresiva, asumiendo que el paciente presenta más bien tristeza o una reacción de estrés ante la relevancia del diagnóstico, y que una vez adaptados a su nueva condición o superadas las complicaciones somáticas, este ánimo bajo debiera desaparecer espontáneamente ${ }^{1,18}$. Por otro lado, muchos pacientes se mostrarían reticentes a consultar por síntomas emocionales debido al estigma social que existe en torno a las patologías mentales, que en este caso se suma al estigma social que rodea a la infección por $\mathrm{VIH}^{30}$.

Adicionalmente, el traslape sintomático que se produce entre depresión y la infección por VIH hace muchas veces difícil la diferenciación diagnóstica. Las alteraciones neurovegetativas y neurocognitivas propias de la depresión también corresponden a síntomas muy frecuentes de encontrar en el contexto de la enfermedad, ya sea como una causa primaria de la infección como por el efecto de enfermedades oportunistas y/o por efectos farmacoló$\operatorname{gicos}^{18,26,41}$. Es así que los pacientes infectados por VIH suelen presentar inapetencia, baja de peso, insomnio o hipersomnia y fatigabilidad, tal como sucede en un episodio depresivo. Por esta razón, es muy importante detallar la forma de presentación de estos síntomas y sospechar una posible depresión a menos que hayan condiciones médicas claras tales como diarrea crónica con o sin síndrome consuntivo, patología tumoral, patología infecciosa sub-aguda o crónica, un síndrome anémico o enfermedades concomitantes tales como hipotiroidismo o insuficiencia adrenal, que expliquen los síntomas somáticos ${ }^{18,26,41}$.

Ante la presencia de alteraciones neurocognitivas, tales como pérdida de memoria, falla en la concentración o enlentecimiento psicomotor, es muy importante plantearse dentro de los diagnósticos diferenciales no sólo una encefalopatía primaria por VIH (caracterizada al inicio precisamente por estas alteraciones), infecciones del sistema nervioso central o procesos expansivos intracerebrales, sino que además sospechar un posible 
episodio depresivo ${ }^{18,30}$. De hecho, según los hallazgos de Gibbie y cols, la baja en el desempeño neurocognitivo mostraría mayor asociación con los síntomas depresivos que con la infección por VIH y sus consecuencias a nivel del sistema nervioso central ${ }^{42}$. El origen de la fatigabilidad resulta particularmente importante de determinar, ya que puede ayudar a dilucidar si un paciente presenta o no depresión. Al respecto, Millikin y cols, realizaron un estudio en pacientes de sexo masculino en el que la fatigabilidad mostró asociación con el diagnóstico de depresión, pero no así con el estado de seropositividad ni con el uso de medicamentos de prescripción frecuente en la infección por $\mathrm{VIH}^{43}$. Por lo mismo, Colibiazzi y cols, preconizan realizar la diferenciación entre fatiga física, fatiga emocional (que estaría ligada a la incapacidad de sentir placer o anhedonia propia de la depresión) y la fatiga mental asociada a déficits neuro-cognitivos ${ }^{18}$.

Los bajos índices de tratamiento psiquiátrico en estos pacientes guardan estrecha relación con el sub-diagnóstico imperante; sin embargo, éste no pareciera ser el único factor involucrado ${ }^{1,30,41}$. En lo que respecta a la administración de medicamentos, se ha planteado que los médicos se mostrarían reticentes a indicar antidepresivos en ciertos pacientes, tales como los de bajo nivel educacional y de mayor edad, las mujeres, y los pertenecientes a ciertos grupos étnicos (p. ej.: afro-americanos), al asumir que no serán adherentes al tratamiento ${ }^{1,30,41}$. Es probable también que muchos médicos que tratan pacientes con infección por VIH no estén actualizados en el manejo de la depresión y, por lo mismo, eviten iniciar tratamientos antidepresivos. Asimismo, los potenciales efectos adversos y el temor a las interacciones medicamentosas harían que tanto médicos como pacientes se muestren reticentes a usar fármacos antidepresivos ${ }^{30}$.

El tratamiento de la depresión en los pacientes infectados por VIH se basa en los mismos principios que el tratamiento de la depresión para la población general u otros subgrupos con enfermedades crónicas, esto es, un enfoque multimodal que combina farmacoterapia, psicoterapia e intervenciones psicosociales. El tratamiento antidepresivo ha demostrado ser altamente efectivo, tanto en el manejo de la sintomatología anímica como en disminuir el riesgo suicida y contribuir a la mejoría del estado inmunológico y adherencia a la TARV, eliminar conductas de riesgo, incluyendo la mejoría en la calidad de vida y en la sobrevida ${ }^{18,29,35,36,38}$. Una buena adherencia al tratamiento antidepresivo favorece, a su vez, una buena adherencia a TARV. Al respecto, Yun y cols, compararon la adherencia a TARV entre pacientes deprimidos, con y sin tratamiento antidepresivo, encontrando una adherencia a TARV (>95\%) de 65 y $35 \%$ respectivamente, siendo esta una diferencia estadísticamente significativa. Al dividir los pacientes entre adherentes y no adherentes al tratamiento antidepresivo, encontraron una adherencia a
TARV de $69 \%$ y $31 \%$, respectivamente, diferencia que también resultó estadísticamente significativa ${ }^{35}$.

Horberg et al, compararon la evolución durante un año de parámetros de laboratorio de pacientes con infección por VIH en TARV que estaban deprimidos, con y sin tratamiento antidepresivo con inhibidores selectivos de la recaptación de serotonina (ISRS), versus controles en TARV que no estaban deprimidos. Encontraron que los primeros tenían una razón de disparidad (odds ratio) significativamente menor de presentar adherencia a TARV superior al $90 \%$ y carga viral menor a 500 copias/ $\mathrm{ml}$, mientras que aquellos pacientes con adherencia a ISRS mayor al $80 \%$, mostraban adherencia a TARV y niveles de supresión de la replicación viral equivalentes a los controles. Consecuentemente, los pacientes deprimidos adherentes al tratamiento con ISRS mostraron un incremento en el nivel de linfocitos CD4+ que fue estadísticamente significativo respecto a los pacientes deprimidos no adherentes ${ }^{29}$.

Considerando que todas las clases de antidepresivos tienen efectos terapéuticos equivalentes, la decisión del fármaco a utilizar va a depender de las posibles interacciones medicamentosas, los efectos secundarios asociados que se deseen potenciar o evitar, y de la disponibilidad de los fármacos en la red asistencial de salud. Es importante informar a los pacientes que el efecto terapéutico de los antidepresivos tarda entre dos y tres semanas en iniciarse, que el tratamiento debe mantenerse por al menos seis meses en forma continuada, que existen posibles efectos adversos asociados y que su eventual suspensión debe ser escalonada ${ }^{18,40,41,44,45}$. En la Tabla 3 se exponen las características principales de las distintas clases de antidepresivos y considerandos adecuados en pacientes con infección por $\mathrm{VIH}^{46}$.

Debido a su buena tolerancia y bajo riesgo de sobredosis, los ISRS son los antidepresivos de elección en los pacientes con infección por VIH. Los efectos secundarios de los fármacos de este grupo consisten en insomnio, ansiedad, agitación, irritabilidad, alteraciones en el apetito, náuseas y disfunción sexual, los cuales, a excepción del último, suelen revertir a las pocas semanas. La fluoxetina, que se caracteriza por una potente inhibición del apetito, tiene una vida media particularmente prolongada, razón por la que ha de ser usada con precaución, especialmente en pacientes con daño renal. La eventual baja de peso asociada a su uso puede llevar falsamente a la idea de progresión de la infección por VIH o a complicaciones de la TARV. La paroxetina, de mayor efecto anticolinérgico que el resto de los ISRS, es poco recomendable ante alteraciones neurocognitivas significativas. Asimismo, su discontinuación abrupta ha de ser evitada, ya que ésta se asocia a lo que se conoce como síndrome de discontinuación, que remeda un cuadro gripal. La sertralina, citalopram y escitalopram tienen perfiles farmacocinéticos 


\section{Tabla 3. Principales fármacos antidepresivos según clase, efectos adversos y_dosis}

\begin{tabular}{|c|c|c|c|c|c|}
\hline Clase & Efectos secundarios & Nombre & Dosis de inicio & Dosis terapéutica & Otros usos \\
\hline \multirow[t]{4}{*}{$\begin{array}{l}\text { Inhibidores selectivos } \\
\text { de recaptación de } \\
\text { serotonina (ISRS) }\end{array}$} & \multirow{4}{*}{$\begin{array}{l}\text { Disfunción sexual }{ }^{1} \text {, síntomas gastrointestinales } \\
\text { (náuseas, diarrea, anorexia' }^{1} \text {, vómitos), aumento o } \\
\text { pérdida de peso, cefaleas', ansiedad }{ }^{1} \text {, insomnio y } \\
\text { sedación }^{2} \text {, sueños vívidos y pesadillas, síntomas extra- } \\
\text { piramidales }{ }^{1} \text {, actividad anticolinérgica leve }{ }^{2} \text {, síndrome } \\
\text { (sd) serotoninérgico, sd discontinuación }{ }^{2} \text {, elevación } \\
\text { prolactina y galactorrea, convulsiones }{ }^{5} \text {, alteración de } \\
\text { la función plaquetaria }{ }^{5} \text {, neutropenia }{ }^{5} \text {, hipoglicemia }{ }^{5} \text {, } \\
\text { hiponatremia }^{5} \text {. }\end{array}$} & Fluoxetina & 20 mg/día (am) & 10-80 mg/día (am) & Bulimia, TOCa \\
\hline & & Paroxetina & 20 mg/día (am) & 10-50 mg/día (am) & $\begin{array}{l}\text { TOC }{ }^{\text {, }} \text { fobia social, tras- } \\
\text { torno de pánico, migraña }\end{array}$ \\
\hline & & Sertralina & 50 mg/día (am) & 50-200 mg/día (am) & TOCa $^{\mathrm{a}}$ TEPT \\
\hline & & Citalopram & 20 mg/día & 20-60 mg/día & $\begin{array}{l}\text { TOCa, neuropatía diabé- } \\
\text { tica, trastorno de pánico }\end{array}$ \\
\hline Tricíclicos & $\begin{array}{l}\text { Actividad anticolinérgica, sedación, hipotensión } \\
\text { ortostática, diaforesis, convulsiones, alteraciones de la } \\
\text { conducción cardiaca, arritmias. }\end{array}$ & Amitriptilina & 25 mg/día (pm) & 50-300 mg/día (pm) & $\begin{array}{l}\text { Dolor crónico, insomnio, } \\
\text { migraña, neuralgia post- } \\
\text { herpética }\end{array}$ \\
\hline \multirow{2}{*}{$\begin{array}{l}\text { Inhibidores selectivos } \\
\text { de recaptación de } \\
\text { serotonina y } \\
\text { noradrenalina (ISRSN) }\end{array}$} & \multirow{2}{*}{$\begin{array}{l}\text { Náuseas, somnolencia, sequedad de boca, mareos, } \\
\text { constipación, astenia, nerviosismo }{ }^{3} \text {, ansiedad }{ }^{3} \text {, ano- } \\
\text { rexia, visión borrosa }{ }^{3} \text {, disfunción sexual }{ }^{3} \text {, sudoración }{ }^{4} \text {, } \\
\text { sd discontinuación }{ }^{3} \text {. }\end{array}$} & Venlafaxina & $37,5 \mathrm{mg} \mathrm{c} / 12 \mathrm{hrs}$ & $75-150 \mathrm{mg} \mathrm{c} / 12 \mathrm{hrs}$ & $\begin{array}{l}\text { TOC, trastorno ansiedad } \\
\text { generalizada, trastorno } \\
\text { de pánico }\end{array}$ \\
\hline & & Duloxetina & 40-60 mg/día & 40-120 mg/día & Neuropatía diabética \\
\hline $\begin{array}{l}\text { Inhibidores de } \\
\text { recaptación } \\
\text { noradrenérgica y } \\
\text { serotoninérgica }\end{array}$ & $\begin{array}{l}\text { Cefalea, insomnio, molestias respiratorias altas, náu- } \\
\text { seas, inquietud, agitación, irritabilidad, sequedad } \\
\text { de boca, constipación, baja de peso, aumento de la } \\
\text { libido, convulsiones (a dosis altas) }\end{array}$ & Bupropión & $50-75 \mathrm{mg} \mathrm{c} / 12 \mathrm{hrs}$ & $\begin{array}{l}150 \mathrm{mg} \mathrm{c} / 12 \mathrm{hrs}- \\
150 \mathrm{mg} \mathrm{c} / 8 \mathrm{hrs}\end{array}$ & $\begin{array}{l}\text { TDAHc, suspensión taba- } \\
\text { co, } \text { TEPT }^{\text {b }}\end{array}$ \\
\hline $\begin{array}{l}\text { Moduladores } \\
\text { serotoninérgicos y } \\
\text { noradrenérgicos }\end{array}$ & $\begin{array}{l}\text { Somnolencia, aumento del apetito, sequedad de boca, } \\
\text { constipación, mareos, mialgias, sueños vívidos, poten- } \\
\text { ciación del efecto sedante del alcohol, elevación del } \\
\text { colesterol y de las transaminasas, neutropenia }{ }^{5} \text {. }\end{array}$ & Mirtazapina & 15 mg/día (pm) & 15-45 mg/día (pm) & $\begin{array}{l}\text { Trastorno ansioso, in- } \\
\text { somnio }\end{array}$ \\
\hline $\begin{array}{l}\text { Moduladores } \\
\text { serotoninérgicos }\end{array}$ & $\begin{array}{l}\text { Somnolencia, hipotensión ortostática, gastritis, pria- } \\
\text { pismo }\end{array}$ & Trazodona & 50 mg/día (pm) & $\begin{array}{l}200 \mathrm{mg} / \mathrm{día}(\mathrm{pm}) \text { - } \\
200 \mathrm{mg} \mathrm{c} / 12 \mathrm{hrs}\end{array}$ & $\begin{array}{l}\text { Trastorno ansioso, trastor- } \\
\text { no de pánico, insomnio }\end{array}$ \\
\hline
\end{tabular}

más favorables que fluoxetina y paroxetina, por lo que su uso es preferible en pacientes $\mathrm{VIH}^{18}$.

A pesar de su efectividad, los antidepresivos tricíclicos (ATC) no se recomiendan, debido a su amplia gama de efectos adversos asociados a sus propiedades anticolinérgicas, tales como hipotensión ortostática, disminución de la capacidad neurocognitiva y delirio, retención urinaria y exacerbación de retardos de conducción eléctrica miocárdica, ante los cuales los pacientes infectados por VIH son particularmente vulnerables. Su uso ha de reservarse para aquellos pacientes asintomáticos de su infección por VIH en quienes se hayan descartado alteraciones neurocognitivas, ya que incluso en pacientes médicamente asintomáticos podrían aparecer signos de demencia. En caso de usarse un ATC, es preferible elegir aquellos con menor efecto anticolinérgico e hipotensor, tales como nortriptilina o desipramina ${ }^{18,40,44,45}$. Por esta causa la amitriptilina, de uso ocasional en el manejo del dolor neuropático asociado a infección por VIH o a su tratamiento, debe usarse con extrema precaución.

Los inhibidores de la monoaminoxidasa (IMAO), ya en desuso en población general, tampoco se recomiendan en los pacientes infectados por VIH. Sus múltiples restricciones alimentarias y el uso concomitante de otros fármacos, los hacen particularmente complejos de utilizar ${ }^{18,44,45}$.

Los inhibidores de la recaptación de serotonina y noradrenalina (ISRSN) son una alternativa eficaz tanto para el manejo de la depresión como del dolor crónico en los pacientes infectados por VIH. La venlafaxina cuenta con un perfil de efectos secundarios similar a los ISRS y, al igual que la paroxetina, no debe ser discontinuada en forma abrupta. A altas dosis se ha asociado a hipertensión diastólica $^{18}$. La duloxetina debe administrarse con precaución en los pacientes con daño hepático o co-infección con virus de hepatitis $\mathrm{C}$, ya que se ha asociado a elevación de las enzimas hepáticas ${ }^{18,45}$. 
El bupropión, antidepresivo con acción noradrenérgica y dopaminérgica, es bien tolerado en los pacientes con infección por VIH, siendo especialmente útil en casos de fatigabilidad marcada debido a su efecto activante. Como disminuye el umbral convulsivante, está contraindicado en patología intracraneal potencialmente inductora de convulsiones (toxoplasmosis, meningitis, accidentes vasculares, tumores cerebrales) y antecedentes de epilepsia. De igual manera, se desaconseja su uso en trastornos alimentarios o desbalances hidroelectrolíticos ${ }^{18}$.

La mirtazapina, antidepresivo noradrenérgico y serotoninérgico específico, presenta un potente efecto antinauseoso y, debido a su perfil antihistamínico se asocia también a sedación, aumento del apetito y ganancia de peso, por lo que podría ser útil en pacientes con síndrome de emaciación asociado ${ }^{18,44,45}$.

Dentro de los antidepresivos atípicos, la nefazodona también destaca por sus propiedades ansiolíticas y sedantes, y al igual que mirtazapina y bupropión presenta mínimos efectos secundarios sobre la función sexual, en comparación con ISRS y SNRIS. Su mayor problema ha sido su posible asociación a hepatotoxicidad e insuficiencia hepática, así como su alto potencial de interacciones medicamentosas. La trazodona, químicamente muy similar a nefazodona, se utiliza principalmente en el manejo del insomnio y como coadyuvante en la terapia con ISRS, siendo sus principales efectos secundarios la hipotensión ortostática y la xerostomía, lo cual limita su uso a pesar de no ser tan frecuentes e intensos como en el caso de los $\mathrm{ATC}^{18}$.

Las benzodiazepinas, usadas por cortos periodos, son útiles en el manejo de los síntomas ansiosos y el insomnio, especialmente mientras se espera la aparición del efecto terapéutico de los antidepresivos. Se recomienda el uso de benzodiazepinas de vida media intermedia y con metabolización simple, como el lorazepam. Esta familia de medicamentos interactúa tanto con inhibidores de proteasa como inhibidores no nucleósidos de la transcriptasa reversa, componentes que se utilizan juntos o separados en prácticamente todas las terapias anti-retrovirales. Su uso continuo no es aconsejado, especialmente en pacientes con antecedentes de abuso de sustancias o daño neuro$\operatorname{cognitivo}^{18,44,45}$.

Si bien suelen ser usados principalmente en el manejo de trastornos maníacos, los estabilizadores del ánimo tales como las sales de litio, lamotrigina y los anticonvulsivantes ácido valproico y carbamazepina, también pueden ser empleados en el tratamiento de los trastornos depresivos. De igual manera, antipsicóticos como clorpromazina o haloperidol pueden ser útiles en el manejo de un paciente deprimido con síntomas psicóticos. Para ambas clases de psicotrópicos se han descrito interacciones medicamentosas con los anti-retrovirales y otros fármacos de uso común en los pacientes infectados por VIH (p.ej.: antimicrobianos). Algunos de estos agentes se asocian a efectos adversos particularmente riesgosos como: síntomas gastrointestinales para el litio o hepatitis grave para ácido valproico, razón por la que los pacientes que requieran su uso deben ser meticulosamente monitoreados ${ }^{18,44,45}$. También han sido utilizados psico-estimulantes como modafilino, metilfenidato y anfetaminas en el manejo de los síntomas depresivos en estos pacientes, mostrando efectividad tanto en el manejo de los síntomas somáticos (falta de energía, fatigabilidad y anorexia), como sobre las alteraciones neuro-cognitivas, el ánimo depresivo y la ideación suicida. Sin embargo, al igual que para las benzodiazepinas, es importante considerar el riesgo de adicción y abuso de estas sustancias ${ }^{11}$. El uso de la terapia de reemplazo androgénica, probada en la recuperación de la masa muscular y la fatiga física ha sido estudiada para el manejo de la sintomatología depresiva. Varios estudios han demostrado efectos positivos tanto de testosterona como de dihidro-epiandrosterona, pero los resultados no han sido concluyentes ${ }^{18,44,45}$.

Shippy y cols, evaluaron la eficacia de s-adenosilmetionina (co-enzima que participa en la reducción de los grupos metilo y para la cual se han descrito efectos antidepresivos), encontrando una reducción significativa de los síntomas depresivos, a la semana de tratamiento ${ }^{24}$. Neidig y cols, evaluaron el efecto de un programa de entrenamiento aeróbico de 12 semanas, reportando una reducción significativa tanto de los síntomas anímicos como somáticos asociados a la depresión ${ }^{41}$. Sustancias de consumo popular con supuestas propiedades antidepresivas, como la hierba de San Juan, no han demostrado efectividad, y más aún, su uso puede ser perjudicial. Dada la marcada interacción con inhibidores de proteasa, está contraindicada su co-administración, por lo que debe evitarse la automedicación de esta sustancia ${ }^{47,48}$. En cuanto a la psicoterapia, en estos pacientes se ha demostrado la efectividad tanto de la terapia interpersonal como de la cognitivo-conductual, particularmente la de tipo grupal $^{18,31}$.

Es muy importante tener claro que tanto los psicotrópicos como algunos de los anti-retrovirales son metabolizados por las isoenzimas del citocromo $\mathrm{P} 450$, lo cual genera un enorme potencial de interacciones medicamentosas ${ }^{49-52}$. Por lo mismo, ante pacientes en TARV que requieran tratamiento farmacológico del cuadro depresivo, es prudente revisar primero las posibles interacciones medicamentosas descritas en manuales ${ }^{47,48}$ o páginas $\mathrm{web}^{53}$. Asimismo, en estos pacientes se recomienda iniciar el tratamiento antidepresivo con dosis más bajas y titulación más lenta, especialmente en los casos de enfermedad avanzada o regímenes medicamentosos complejos, de igual forma que se debe hacer el ajuste de dosis para evitar la aparición de efectos secundarios indeseables ${ }^{1,40,44,45}$.

No todas las interacciones entre psicotrópicos y 
anti-retrovirales tienen traducción clínica ni están todas dilucidadas o estudiadas, especialmente para los antidepresivos y los anti-retrovirales de más reciente aparición. A pesar de esto, existen ciertas interacciones descritas que han de tenerse en cuenta. Por ejemplo, entre los inhibidores nucleósicos de transcriptasa reversa de uso actual (zidovudina, lamivudina, abacavir, didanosina y tenofovir) destaca la interacción entre zidovudina y ácido valproico, lo que determina un uso prudente de la co-medicación ${ }^{47,48}$. Entre los inhibidores no nucleósicos de la transcriptasa reversa de uso actual (efavirenz y nevirapina), destaca la interacción negativa de efavirenz con las benzodiazepinas midazolam, triazolam y alprazolam, y con la hierba de San Juan, por lo que su coadministración está contraindicada ${ }^{47,48}$. Asimismo, se ha visto que los niveles plasmáticos de carbamazepina y otros anticonvulsivantes disminuyen con este anti-retroviral, lo que pudiera disminuir el efecto de los primeros ${ }^{47,48}$. Los inhibidores de proteasa (indinavir, atazanavir, lopinavir, darunavir, saquinavir, tipranavir, fosamprenavir y, en especial, ritonavir), que se caracterizan por inducir la reducción marcada de las concentraciones plasmáticas de otros fármacos, pudiendo verse también disminuidas sus propias concentraciones plasmáticas, representan el mayor riesgo de interacción con los fármacos usados en el tratamiento antidepresivo. Los con mayor susceptibilidad de interacciones son carbamazepina y clonazepan (con menor intensidad con atazanavir y tripanavir que con el resto), triazolam, midazolam y alprazolam ${ }^{47,48}$. También se han observado aumentos variables de los tricíclicos y de los ISRS, que obligan a evaluar la compatibilidad de este grupo de anti-retrovirales con cada fármaco antidepresivo en particular ${ }^{47,48}$.

Por otro lado, es importante tener en cuenta que para todas las clases de anti-retrovirales se han descrito efectos adversos neuro-psiquiátricos. Estos incluyen alteraciones neuro-cognitivas, trastornos del sueño, cuadros psicóticos o maníacos, depresión grave e ideación suicida, entre otros $^{26,32,33}$. Estos efectos son particularmente frecuentes y clínicamente relevantes para efavirenz, describiéndose hasta 50 a $70 \%$ de incidencia al inicio de terapias que incluyen este fármaco, predominando mareos, alteraciones del sueño, sueños vividos, confusión y sensación de despersonalización. Si bien los efectos suelen ser leves y transitorios, en algunos casos la sintomatología neuro-psiquiátrica asociada puede ser de tal magnitud y/o duración que ha de considerarse el cambio e incluso la suspensión temporal de la TARV ${ }^{26,32,33}$. Es por esto que Treisman y Kaplin sugieren que, de haber antecedentes de depresión grave, ideación suicida o abuso de sustancias, se inicie el tratamiento profiláctico para estos cuadros previo al inicio de TARV. Asimismo, sugieren que en aquellos con un episodio depresivo actual y que requieren inicio de TARV, se debe iniciar el tratamiento antidepresivo lo antes posible, considerando el uso de anti-retrovirales con menores efectos secundarios neuro-psiquiátricos; en particular efavirenz ${ }^{26}$.

Finalmente, es importante considerar que, si bien el tratamiento antidepresivo en los pacientes con infección por VIH puede ser iniciado y monitorizado por cualquier médico clínico, cuando la sintomatología depresiva es grave, hay gran discapacidad social y/o laboral, ideación suicida persistente o intentos suicidas, presencia de síntomas psicóticos, sospecha de trastorno bipolar y/o abuso de sustancias en forma concomitante, es importante que la evaluación y el manejo se realice en forma coordinada con el psiquiatra. Lo mismo se recomienda para pacientes que han tenido una mala respuesta al tratamiento antidepresivo $^{18}$.

\section{Conclusiones}

La depresión es uno de los principales trastornos psiquiátricos que aquejan a los pacientes con infección por VIH y su prevalencia, descrita es en torno al 37\%, dos a tres veces más alta que en la población general. Es más frecuente en mujeres y adolescentes, y en pacientes con malas redes de apoyo social, asociándose a más rápida progresión de la enfermedad y menor adherencia a TARV. El tratamiento antidepresivo ha mostrado beneficios en el manejo de la sintomatología depresiva propiamente tal, así como en la adherencia a TARV y el consiguiente éxito inmunológico y clínico.

A pesar de esto, la depresión suele ser sub-diagnosticada y sub-tratada lo cual se debe tanto a la reticencia por parte de los pacientes a revelar su sintomatología depresiva como de los médicos clínicos a consultar por la misma, a la dificultad en distinguir el cuadro de las complicaciones propias del VIH y a la poca experiencia que existe en tratar la depresión en los pacientes con infección por VIH. Por lo mismo, es imperativo que los profesionales de la salud que trabajan con pacientes infectados por el VIH estén atentos frente a este problema e incorporen en su práctica clínica la búsqueda activa de síntomas depresivos.

Una estrategia posible de utilizar sería el tamizaje periódico de síntomas depresivos mediante algún instrumento estandarizado, tal como el Inventario de depresión de Beck (BDI) ${ }^{54}$ o la Escala de depresión del Centro de Estudios Epidemiológicos (CES-D) ${ }^{55}$, que han sido ampliamente utilizados en pacientes infectados por VIH, lo que permite identificar a aquellos pacientes que requieren mayor evaluación y eventual tratamiento antidepresi$\mathrm{vo}^{3,7,12,22,25,27,56}$. Es esencial que los médicos clínicos que tratan pacientes seropositivos para VIH se familiaricen con el diagnóstico de la depresión, conozcan el enfoque terapéutico, manejen el tratamiento inicial, estén al tanto 
de las posibles interacciones medicamentosas entre psicotrópicos y anti-retrovirales y conozcan los criterios de derivación a especialistas en psiquiatría. Idealmente, los servicios de salud mental debieran estar ubicados en los mismos centros en los cuales los pacientes reciben sus controles periódicos de salud, pero de no ser ése el caso, al menos ha de asegurarse un acceso expedito a los mismos.

\section{Resumen}

La depresión es una de las principales co-morbilidades psiquiátricas en el curso de la infección por VIH, presentándose con una prevalencia significativamente mayor que en población general (alrededor de 35\%). Su presencia se ha asociado a deterioro de la calidad de vida, progresión de la enfermedad por VIH y disminución en la adherencia a la terapia anti-retroviral. El adecuado tratamiento antidepresivo ha demostrado ser efectivo en el manejo de la sintomatología depresiva, en la mejoría de parámetros clínicos y de laboratorio, y en reforzar la adherencia a la terapia anti-retroviral. A pesar de su importancia, la depresión suele ser sub-diagnosticada y sub-tratada en estos pacientes. En este trabajo se revisan los principales hallazgos internacionales sobre prevalencia, factores de riesgo, consecuencias y abordaje de la depresión en personas infectadas por VIH.

\section{Refrencias}

1.- Asch S, Kilbourbe A, Gifford A, Burnam M, Turner B, Shapiro M, et al. Underdiagnosis of HIV. Who are we missing? J Gen Inter Med 2003; 18: 450-60.

2.- Morrison M, Petitto J, Ten Have T, Gettes D, Chiappinni M, Weber A, et al. Depressive and anxiety disorders in women with HIV infection. Am J Psychiatry 2002; 159: 789-96.

3.- Weiser D, Riley E, Ragland K, Hammer G, Clark R, Bansberg D. Brief report: Factors associated with depression among homeless and marginally housed HIV-infected men in San Francisco. J Gen Intern Med 2006; 21: 61-4.

4.- Rabin J. HIV and depression: 2008 review and update. Curr HIV/AIDS Rep 2008; 5:163-71.

5.- Bing E, Burnam A, Longshore D, Fleishman J, Donald C, London A, et al. Psychiatric disorders and drug use among human immunodeficiency virus infected adults in the United States. Arch Gen Psychiatry 2001; 58: 721-8.

6.- Pence B, Miller W, Whetten K, Eron J, Gaynes B. Prevalence of DSM-IV mood, anxiety and substance use disorders in an HIV clinic in the Southeastern United States. J Acquir Immun Defic Syndr 2006; 42: 298-306.

7.- Jin H, Hamptom Atkinson J, Yu X, Heaton R, Shi C, MarcotteT, et al. Depression and suicidality in China. J Affect Disord 2006, 94: 269-75.

8.- Noqueira L, De Fátima P, Crosland M. Anxiety and depression assessment prior to initiating antiretroviral treatment in Brazil. AIDS Care 2006; 18: 529-36.

9.- Antakly de Mello V, Malgebier A. Depression in women infected with HIV. Rev Bras Psiquiatr 2006; 28: 10-7.

10.- Gibbie T, Hay M, Hutchison C, Mijch A. Depression, social support and adherence to highly active antirretroviral therapy in people living with HIV/AIDS. Sex Health 2007; 4: 227: 32.

11.- Pao M, Lyon M, D'Angelo L, Schuman W,
Tipnis T, Mrazak D. Pychiatric diagnoses in adolescents seropositive for the human immunodeficiency virus. Arch Pediatr Adolesc Med 2000; 154: 240-4.

12.- Israelski D, Prentiss D, Lubega S, Balmas G, García P, Muhammad M,et al. Psychiatric co-morbidity in vulnerable populations receiving primary care for HIV/AIDS. AIDS Care 2007; 19: 220-25.

13.- Turner B, Fleischmann J. Effect of dysthymia on receipt of HAART by minority HIV-infected women. J Gen Intern Med 2006; 21: 1235-41.

14.- Rakbin J, Ferrando S, Jacobsberg L, Fishman B. Prevalence of axis I disorders in an AIDS cohort: a cross-sectional, controlled study. Compr Psychiatry 1997; 38: 146-54.

15.- Berger-Greenstein J, Cuevas C, Brady S, Trezza G, Richardson M, Keane T. Mayor depression in patients with HIV/AIDS and substance abuse. AIDS Patient Care STDS 2007; 21: 942-55.

16.- Olley B, Gxamza F, Seedat S, Theron H, Taljaard J, Reid E, et al. Psychopathology and coping in recently diagnosed HIV/AIDS patients- the role of gender. S Afr Med J 2004; 94: 500 .

17.- Olley B, Zeir M, Seedat S, Stein D. Post-traumatic stress disorder among recently diagnosed patients with HIV/AIDS in South Africa. AIDS Care 2005; 17: 550-7

18.- Colibazzi T, Hsu T, Gilmer S. Human immunodeficiency virus and depression in primary care: A clinical review. Prim Care Companion J Clin Psychiatry 2006; 8: 201-11.

19.- Judd F, Komiti A, Chua P, Mijch A, Hoy J, Grech P, et al. Nature of depression in patients with HIV/AIDS. Aust NZ J Psychiatry 2005; 39: 826-32.

20.- Rush A, Keller M, Bauer M, Dunner D, Frank E, Klein D (Grupo de trabajo para los trastornos del estado del ánimo). Trastornos del estado del ánimo. En: Pichot, López-Ibor, Valdés (Editores).Manual diagnóstico y estadístico de los trastornos mentales (DSM IV), Masson S.A,
Barcelona, 1995.

21.- Turner B, Laine C, Cosler L, Hauck W. Relationship of gender and health care delivery with antiretroviral adherence in HIV- infected drug users. J Gen Intern Med 2003; 18: 248-57.

22.- Cook J, Grey D, Burke J, Cohen M, Gurtman A, Richardson J, et al. Depressive symptoms and AIDS- related mortality among a multisite cohort of HIV-positive women. Am J Public Health 2004; 94: 1133-40.

23.- Cook J, Grey D, Burke-Miller J, Cohen M, Vlahov D, Kapadia F, et al. Illicit drug use, depression and their association with highly active antiretroviral therapy in HIV-positive women. Drug Alcohol Depend 2007; 89: 74-81.

24.- Olley B, Seedat S, Nei D, Stein D. Predictors of mayor depression in recently diagnosed patients with HIV/AIDS in South Africa. AIDS Patients Care STDS 2004; 18: 481-487.

25.- Yi M, Mrus J, Wade T, Ho M, Hornung H, Cotton S, et al. Religion, spirituality, and depressive symptoms in patients with HIV/AIDS. J Gen Intern Med 2006; 21: s21-s27.

26.- Treisman G, Kaplin A. Neurologic and psychiatric complications of antiretroviral agents. AIDS 2002; 16: 1201-15.

27.- Lima V, Geller J, Bangsberg D, Patersson T, Daniel M, Kerr T,et al. The effect of adherence on the association between depressive symptoms and mortality among HIV-infected individuals first initiating HAART. AIDS 2007; 21: $1175-83$.

28.- Treisman G, Angelino A. Interrelation between psychiatric disorders and the prevention and treatment of HIV infection. Clin Infect Dis 2007; 45: s313-s317.

29.- Horberg M, Silverberg M, Hurley L, Towner W, Klein D, Bersoff-Matcha S, et al. Effects of depression and selective serotonin reuptake inhibitor use on adherence to highly active antiretroviral therapy and on clinical outcomes in HIV- infected patients. J Acquir Immune Defic Syndr 2008; 47: 384-90.

30.- Shippy R, Mendez D, Jones K, Cergnul I, 
Karpiak S. S- adenosylmethionine (SAM-E) for the treatment of depression in people living with HIV/AIDS. BMC Psychiatry 2004; 4:38. doi:10.1186/1471-244X-4-38. Disponible en: http://www.biomedcentral.com/1471244X/4/38.

31.- Olantunji B, Mimiaga M, O'Cleirigh, Safren S A review of treatment studies of depression in HIV. Top HIV Med 2006; 14: 112-24.

32.- Raines C, Radcliffe O, Treisman G. Neurologic and psychiatric complications of antiretroviral agents, J Assoc Nurses AIDS Care 2005; 16; $35-48$.

33.- Cespedes M, Aberg J. Neuropsychiatric complications of antiretroviral therapy. Drug Saf 2006; 29: 865-74.

34.- Di Iorio C, Mc Carty F, De Padilla L, Resnicow K, Mc Donell M, Yeager K, et al. Adherence to antirretroviral medication regimens: A test of a psychosocial model. AIDS Behav 2009; 13 : 10-22.

35.- Yun L, Maravi M, Kobayashi J, Barton P, Davidson A. Antidepressant treatment improves adherente to antirretroviral therapy among depressed HIV-infected patients. J Acquir Immune Defic Syndr 2005; 38: 432-8.

36.- Dalessandro M, Conti C, Gambi F, Falasca K, Doyle R, Conti P, et al. Antidepressant therapy can improve adherence to antiretroviral therapy regimens among HIV-infected and depressed patients. J Clin Psychofarmacol 2007; 27: 5861.

37.- Applebaum A, Richardson M, Brady S, Brief D, Keane T. Gender and other psychosocial factors as predictors of adherence to highly active retroviral therapy in adults with comorbid HIV/AIDS, psychiatric and substance related disorder. AIDS Behav 2009; 13: 60-5.

38.- Starece F, Ammassari A, Trotta M, Murri R,
De Longis $\mathrm{P}$, Izzo C,et al. Depression is a risk factor for suboptimal adherence to highly active antiretroviral therapy. J Acquir Immune Defic Syndr 2002; 15: s136-s139. A

39.- Ammassari A, Antinori A, Aloisi M, Trotta M, Murri R, Bartoli L, et al. Depressive symptoms, neurocognitive impairment, and adherence to highly active antiretroviral therapy among HIV-infected persons. Psychosomatics 2004; 45: 394-402.

40.- Thompson A, Silverman D, Dzeng L, Treisman G. Psychotropic medications and HIV. Clin Infect Dis 2006; 42: 1305-10.

41.- Neidig J, Smith B, Brashers D. Aerobic exercise training for depressive symptom management in adults living with HIV infection. J Assoc Nurses AIDS Care 2003; 14: 30-40.

42.- Gibbie T, Mijch A, Ellen S, Hoy J, Hutchinson $\mathrm{C}$, Wright E, et al. Depression and neurocognitive performance in individuals with HIV/AIDS: 2 year follow-up. HIV Med 2006; 7: 112-21.

43.- Millikin C, Rourke S, Halman, Power C. Fatigue in HIV/AIDS is associated with depression and subjective neurocognitive complaints but not neurophysiological funtioning. J Clin Exp Neuropsychol 2003; 25: 201-15.

44.- Cruess D, Evans D, Repetto M, Gettes D, Douglas S, Petitto J. Prevalence, diagnosis, and pharmacological treatment of mood disorders in HIV disease. Biol Psychiatry 2003; 54: 307-16.

45.- Repetto M, Petitto J. Psychopharmacology in HIV-infected patients. Psychosom Med 2008; 70: 585-92.

46.- Sadock B, Alcott V. Terapias Biológicas. Cancro $\mathrm{R}$, editor consultor principal. Kaplan- Sadock: Sinopsis de Psiquiatria, Ciencias de la conducta/ Psiquiatría clínica. Novena edición.Buenos Aires:Waverly Hispánica S.A; 2004; 974-1150.
47.- Bartlett JG, Gallant JE. Medical Management of HIV Infection. John Hopkins Medicine Health Publishing Business Group, Baltimore, MA, 2005.

48.- Sande MA, Eliopoulos GM, Moellering RC and Gilbert DN (editors). The Sanford Guide to HIV/AIDS Therapy 2008. Antimicrobial Therapy, INC, Sperryville, VA. $16^{\text {th }}$ edition, 2008

49.- Greenblatt D, Von Moltke L, Harmatz J, Fogelman S, Chen G, Graf J, et al. Short-term exposure to low-doseritonavir impairs clearance and enhances adverse effects of trazodone. J Clin Pharmacol 2003; 43: 414-22.

50.- Serotonin syndrome in HIV-infected individuals receiving antiretroviral therapy and fluoxetine. AIDS; 2001: 1281-85.

51.- Levin G, Nelson L, DeVane C, Preston S, Eisele G, Carson S. Psychopharmacol Bull 2001; 35: 62-71.

52.- Gutierrez G, Rosenberg J, Abramowitz W. An evaluation of the potential pharmacokinetic interaction between escitalopram and the cytochrome P450 3A4 inhibitor ritonavir. Clin Ther 2003; 25: 1200-10.

53.- Interaction charts. Disponible en: http://www.hivdruginteractions.org/. (accedido el 31 mayo 2009).

54.- Beck AT, Ward CH, Mendelson M, Mock J, Erbaugh J. An inventory of measuring depression. Arch Gen Psychiatry 1961; 4: 561-71.

55.- Beck AT, Steer RA. Beck Depression Inventory (BDI). En: American Psychiatric Association (ed). Handboock of Psychiatric Measures. Washington DC, USA. 2000. Pp. 519-523.

56.- Bouhnik A, Préau M, Vincent E, Carrieri M, Gallais H, Lepeu G, et al. Depression and clinical progression in HIV-infected drug users treated with highly active antiretroviral therapy. Antivir Ther 2005; 10: 53-61. 